\title{
Herpes zoster vaccine: a shot to maintain wellbeing
}

\author{
Jean-Pierre Michel
}

Received: 10 November 2014/ Accepted: 10 November 2014/Published online: 14 January 2015

(C) Springer International Publishing Switzerland 2015

The current era is full of contradictory vaccine issues. The emerging Ebola pandemic stimulates the world's citizens who are calling for the urgent development of vaccine to prevent the disease [1]. They seem to be suddenly remembering that vaccines represent a major advancement in public health [2]. While the complete eradication of polio, neonatal tetanus, congenital rubella, and measles are still the main targets in less developed countries [3], inhabitants of high-resource countries are focusing on rare adverse effects of vaccines to discredit the efficacy and effectiveness of vaccination. As a result, there is an increase in vaccine-preventable diseases in both children and adults. Indeed, it has been estimated that ten times more adults (40 to 50 thousand) die than children in the USA annually from potentially vaccine-preventable diseases [4].

In this controversial context, alongside vaccines for lifethreatening diseases, there have emerged new vaccines to prevent the occurrence of diseases and their complications. Herpes zoster (HZ) vaccine [5-7], is the best example of such quality improvement vaccines. In this issue, Stefania Maggi and collaborators present a comprehensive and upto-date review of how the $\mathrm{HZ}$ vaccine improves healthy ageing [8]. This paper raises some questions concerning the global epidemiology of the disease, improved knowledge of $\mathrm{HZ}$ risk factors, the well-known and more surprising consequences of the disease, as well as the increasing dilemma about age and the new indications for the currently available vaccine.

J.-P. Michel ( $\square)$

Geneva Medical School, Geneva University, 40 A route de Malagnou, Geneva 1208, Switzerland

e-mail: Jean-Pierre.Michel@unige.ch
The incidence rate (IR) of $\mathrm{HZ}$ in Italy, at 6.3 per thousand person-years $[8,9]$, is higher than the mean incidence rate of 3-5 per thousand person-years reported in an analysis of 130 studies conducted in 26 countries in North America, Europe, and Asia-Pacific [10]. This could be related to a temporal increase in the incidence of $\mathrm{HZ}$, often occurring before the introduction of varicella vaccination programmes [11], as in Finland [11] and Germany [12]. In the United Kingdom, one study reported that the median age at $\mathrm{HZ}$ diagnosis was 62 years (interquartile range 48-73) [13]. The IR was more or less stable between the ages of 10 and 44 years (at around 2.4 per thousand personyears), but increased more than threefold, to reach 15 in the 80-84 year old age group [12]. In the same study population, the IR was $36 \%$ higher in females than in males [12]. Prodromic pain was experienced by $69.5 \%$ of South Korean patients, of whom $95.1 \%$ had moderate-to-severe pain [14]. In Italy, postherpetic neuralgia (PHN) occurred in 20.6 and $9.2 \%$ of $\mathrm{HZ}$ patients older than 50 years after 3 and 6 months respectively [8,15], which corresponds to the mean 6-month PHN risk reported in the world literature [16].

The risk factors for $\mathrm{HZ}$ have recently been better identified, and Maggi's paper describes them in detail [8]. However, it is important to insist on a few of these factors. In a study cohort of 144,959 adults diagnosed with herpes zoster between 2000 and 2011, severely immunosuppressive conditions represented the main risk (with odds ratios between 1.78 and 13.46), followed by systemic lupus erythematous, rheumatoid arthritis and chronic pulmonary disease (increased risk over $30 \%$ ) [13]. In the same study, it appeared that asthma, chronic kidney disease, and depression increased the risk of $\mathrm{HZ}$ by $10 \%$, while no association was found with diabetes [13]. Stress and stressful life events were also found to be antecedents to 
outbreaks of HZ [17]. Similarly, the development of PHN has been associated with the psychological antecedents of somatization, nonspecific personality psychopathology, hypochondriasis, and somatic symptoms [17]. However, explicit psychiatric diagnoses, mood, and anxiety disorders have rarely been examined, except for depression, with $\mathrm{HZ}$ reported to be an independent risk factor for major depression and depressive disorders in a large prospective study [18]. In general, the relative effects of these risk factors for $\mathrm{HZ}$ onset decreased with increasing age [13].

Interestingly, recent studies have reported that chronic cytomegalovirus (CMV) infection may stimulate increasing $\mathrm{HZ}$ virus reactivation with age [19], and that there is a significant increase in chronic fatigue after HZ, with incidence increasing over time [20]. It is impossible not to pose the critical question of whether chronic activation of inflammatory pathways plays a role in the pathogenesis of frailty [21]. Indeed, the relationship between chronic CMV infection and HZ may compound the risk frailty [22]. Moreover, HZ and its complications may impair physical, social, and mood function, frequently provoking functional decline [23]. This in turn leads to a decrease in general or usual activity and self-care, associated with mobility disturbances, reduced mood and enjoyment [18], as well as disturbed sleep and relations with others [24, 25]. The repercussions on daily life of $\mathrm{HZ}$, and even more so of $\mathrm{PNH}$, negatively impact on patients and their family members, who express feelings of helplessness, frustration, sadness, and anger [26].

Recent convincing evidence appears to associate $\mathrm{HZ}$ infection, particularly $\mathrm{HZ}$ opthamicus, with an increased risk of stroke within 6 months [27]. A retrospective study of 106,601 HZ cases and 213,202 age- and gender-matched controls demonstrated that $\mathrm{HZ}$ is an independent risk factor for vascular diseases in the UK population [28]. Adjusted hazard ratios [95\% confidence intervals] reached 1.15 [1.09-1.21] for transient ischemic attack (TIA), and 1.10 [1.05-1.16] for myocardial infarction. The risk of TIA, stroke, and myocardial infarction increased significantly if $\mathrm{HZ}$ occurred in patients less than 40 years of age. In older subjects, better ascertainment of vascular risk factors and early intervention may explain the reduction in risk of stroke after HZ [28]. The so-called "varicella zoster virus (VZV) vasculopathy" is probably directly linked to VZV infection and replication within arterial walls, provoking vascular damage and disruption of vascular flow [29]. According to Nagel et al. [30] "VZV vasculopathy" could lead to transient ischemic attacks and ischemic and haemorrhagic stroke, but also temporal artery infection mimicking giant cell arteritis, extra cranial vasculopathy, aneurysm with and without subarachnoid haemorrhage, as well as spinal cord infarction and peripheral thrombotic disease.
All the possible consequences of $\mathrm{HZ}$ mentioned above adequately explain the results of a large prospective study proving that major depression and depressive symptoms occur more in $\mathrm{HZ}$ patients than in controls (respectively, 2.2 vs. $1.4 \%$ and 4.3 vs. $3.2 \%$ ) [18].

Despite its established efficacy against HZ and PHN, well summarized in Maggi's paper (HZ vaccine effectiveness is $48 \%$ for $\mathrm{HZ}$ and $59 \%$ for PHN at 3 months [27]), the uptake of $\mathrm{HZ}$ vaccine remains extremely low, at only $3.9 \%$ of eligible subjects according to Langan et al. [27], and only $16 \%$ in 2011 [31] and $20 \%$ in 2012 for US adults over 60 years of age [32].

However, the most surprising of all these considerations is that the patients at greatest risk (i.e. those with severely immunosuppressive conditions and individuals below the age of 40 years at risk of vascular events) are not currently eligible to receive the vaccine [13].

Due to the increased severity of varicella in immunocompromised subjects, and in light of a recent prospective study demonstrating that $\mathrm{HZ}$ vaccine prior to chemotherapy reduced HZ incidence by half [33], Oxman et al. [34] purport that it may be time to reconsider the use of $\mathrm{HZ}$ vaccine in selected populations of immunocompromised patients. Updated HZ recommendations published by the Strategic Advisory Group of Experts (SAGE) in May 2014 have already widened the HZ recommendations to cover: (a) clinically stable HIV-infected children or adults with $\mathrm{CD} 4+\mathrm{T}$ cell determinations above $15 \%$, including those receiving highly active antiretroviral therapy (HAART); (b) non-immunocompromised individuals with a history of acute lymphocytic leukaemia; and (c) patients with certain solid tumours who have successfully completed chemotherapy and are unlikely to relapse can receive the vaccine at least 3 months after all chemotherapy is completed. Furthermore, the vaccine can safely be given to subjects with isolated defects in antibody production and those with immunodeficiency conditions that include defects in cell-mediated immunity [35]. Indeed, it is also recommended that $\mathrm{HZ}$ vaccination of health care workers in contact with these immunocompromised patients be included in the routine immunization schedule [36].

Nonetheless, as also stressed by Oxman, specific vaccine recommendations should not divert attention from the urgent need to increase the currently inadequate uptake of $\mathrm{HZ}$ vaccine by adults [34]. The debate about the age at which adults should get the $\mathrm{HZ}$ vaccine is still wide open. In the UK, $45 \%$ of $\mathrm{HZ}$ cases occurred before the age of 60 and $65 \%$ in those under 70 [13]. Moreover, as the risk of developing $\mathrm{HZ}$ vasculopathy was proven to be major for subjects under 40 of age [28], the role of $\mathrm{HZ}$ vaccine in younger individuals could be promoted, as well as universal childhood varicella vaccination [37]. 
Acknowledgments The author would like to acknowledge Fiona Ecarnot for her assistance with preparation of this manuscript.

Conflict of interest None.

\section{References}

1. WHO (2014) Glimmers of hope on the Ebola front. Bull World Health Organ 92:704-705

2. Andre FE et al (2008) Vaccination greatly reduces disease, disability, death and inequity worldwide. Bull World Health Organ 86(2):140-146

3. WHO, Global vaccine action plan, Strategic Advisory Group of Experts on Immunization-assessment report 2013, in http:// www.who.int/immunization/global_vaccine_action_plan/OMSIVB-AssessmentReport-20131212v5.pdf, WHO (ed), WHO, Geneva

4. Program, NI (2000) Strategic plan 2000-2005; a blueprint for sustained success. http://www.immregistries.org/resources/NIP strategic_plan.pdf

5. Johnson RW (2009) Herpes zoster and postherpetic neuralgia: a review of the effects of vaccination. Aging Clin Exp Res 21(3):236-243

6. Lang P-O, Michel J-P (2011) Herpes zoster vaccine: what are the potential benefits for the ageing and older adults? Eur Geriatr Med 2(3):134-139

7. Johnson RW, Rice AS (2014) Clinical practice. Postherpetic neuralgia. N Engl J Med 371(16):1526-1533

8. Maggi S, Gabutti G, Franco E et al (2014) Preventing and managing herpes zoster: key actions to foster healthy aging. Aging Clin Exp Res. doi:10.1007/s40520-015-0314-7

9. Gialloreti LE et al (2010) Epidemiology and economic burden of herpes zoster and post-herpetic neuralgia in Italy: a retrospective, population-based study. BMC Infect Dis 10:230

10. Kawai K, Gebremeskel BG, Acosta CJ (2014) Systematic review of incidence and complications of herpes zoster: towards a global perspective. BMJ Open 4(6):e004833

11. Poletti $P$ et al (2013) Perspectives on the impact of varicella immunization on herpes zoster. A model-based evaluation from three European countries. PLoS One 8(4):e60732

12. Hillebrand $\mathrm{K}$ et al. (2014) Incidence of herpes zoster and its complications in Germany, 2005-2009. J Infect. doi:10.1016/j. jinf.2014.08.018

13. Forbes HJ et al (2014) Quantification of risk factors for herpes zoster: population based case-control study. BMJ 348:g2911

14. Song $\mathrm{H}$ et al (2014) Burden of illness, quality of life, and healthcare utilization among patients with herpes zoster in South Korea: a prospective clinical-epidemiological study. Int J Infect Dis 20:23-30

15. Franco E et al (2013) Proportion of post herpetic neuralgia among patients with herpes zoster in Italy-a multicenter prospective observational study. Eur Geriatr Med 4:S115-S116

16. Kawai $\mathrm{K}$ et al (2014) Cost-effectiveness of vaccination against herpes zoster and postherpetic neuralgia: a critical review. Vaccine 32(15): $1645-1653$

17. Sansone RA, Sansone LA (2014) Herpes zoster and postherpetic neuralgia: an examination of psychological antecedents. Innov Clin Neurosci 11(5-6):31-34
18. Chen MH et al (2014) Risk of depressive disorder among patients with herpes zoster: a nationwide population-based prospective study. Psychosom Med 76(4):285-291

19. Ogunjimi B et al (2014) Serology indicates cytomegalovirus infection is associated with varicella-zoster virus reactivation. J Med Virol 86(5):812-819

20. Tsai SY et al (2014) Increased risk of chronic fatigue syndrome following herpes zoster: a population-based study. Eur J Clin Microbiol Infect Dis 33(9):1653-1659

21. Lang PO (2010) et al Immunological pathogenesis of main agerelated diseases and frailty: role of immunosenescence. Eur Geriatr Med 1(2):112-120

22. Wang GC et al (2010) Cytomegalovirus infection and the risk of mortality and frailty in older women: a prospective observational cohort study. Am J Epidemiol 171(10):1144-1152

23. Gavazzi $G$ et al (2014) Impact of herpes zoster on age-related decline in function and adaptation capacity: confrontation of expert opinions. Eur Geriatr Med 5:S76

24. Oster G et al (2005) Pain, medication use, and health-related quality of life in older persons with postherpetic neuralgia: results from a population-based survey. J Pain 6(6):356-363

25. Drolet $M$ et al (2013) Vaccination against herpes zoster in developed countries: state of the evidence. Hum Vaccin Immunother 9(5):1177-1184

26. Lukas K, Bertrand I, Glogge A (2014) The societal impact of herpes zoster and post herpetic neuralgia on patients and family members involved in their care in Germany. Eur Geriatr Med 5:S146-S147

27. Langan SM et al (2014) Risk of stroke following herpes zoster: a self-controlled case-series study. Clin Infect Dis 58(11):1497-1503

28. Breuer J et al (2014) Herpes zoster as a risk factor for stroke and TIA: a retrospective cohort study in the UK. Neurology 82(3):206-212

29. Nagel MA (2014) Varicella zoster virus vasculopathy: clinical features and pathogenesis. J Neurovirol 20(2):157-163

30. Nagel MA, Gilden D (2014) Update on varicella zoster virus vasculopathy. Curr Infect Dis Rep 16(6):407

31. Williams WW, Lu PJ, O'Halloran A, Bridges CB, Pilishvili T, Hales CM, Markowitz LE; Centers for Disease Control and Prevention (CDC) (2014) Noninfluenza vaccination coverage among adults - United States, 2012. MMWR Morb Mortal Wkly Rep 63(5):95-102

32. Hales CM et al (2014) Update on recommendations for use of herpes zoster vaccine. MMWR Morb Mortal Wkly Rep 63(33):729-731

33. Tseng HF et al (2014) Vaccination against zoster remains effective in older adults who later undergo chemotherapy. Clin Infect Dis 59(7):913-919

34. Oxman MN, Schmader KE (2014) Editorial commentary: zoster vaccine in immunocompromised patients: time to reconsider current recommendations. Clin Infect Dis 59(7):920-922

35. (SAGE), S.A.G.o.E. (2014) Meeting of the Strategic Advisory Group of Experts on immunization-conclusions and recommendations. Wkly Epidemiol Rec 2014(89):221-236

36. Weekly epidemiological record Meeting of the Strategic Advisory Group of Experts on immunization, April 2014 - conclusions and recommendations. Weekly epidemiological record Relevé épidémiologique hebdomadaire 2014. http://www.who. int/wer/2014/wer8921.pdf227-9

37. Chapman AL (2014) Herpes zoster and stroke: implications for therapy and vaccination. Clin Infect Dis 59(8):1185 